\title{
Delay-constrained Achievable Rate Maximization in Wireless Relay Networks with Causal Feedback
}

\author{
James C. F. Li and Subhrakanti Dey \\ ARC Special Research Centre for Ultra-Broadband Information Networks (CUBIN) \\ Department of Electrical and Electronic Engineering \\ University of Melbourne, Victoria 3010, Australia \\ Email: c.li, sdey@ee.unimelb.edu.au
}

\begin{abstract}
Motivated by delay-sensitive information transmission applications, we propose an expected achievable rate maximization scheme with a K-block delay constraint on data transmission using a three node cooperative relay network assuming a block fading channel model. Channel information is fed back to the transmitter only in a causal fashion, so that the optimal power allocation strategy is only based on the current and past channel gains. We consider the two simplest schemes for information transmission using a three node (a source, a relay and a destination) relay network, namely the amplify and forward (AF) and decode and forward (DF) protocols. We use a dynamic programming based methodology to solve the (K-block delay constrained) expected capacity maximization problem with a short term (over $\mathrm{K}$ blocks) sum power (total transmission power of the source and the relay) constraint. Furthermore, two simple power allocation schemes for high and low SNR situations are proposed. Extensive numerical results are presented for Rayleigh fading channels, including results demonstrating the accuracy of the high/low SNR approximation based power allocation schemes.
\end{abstract}

\section{INTRODUCTION}

Relay networks were first proposed in [1], and capacity bounds for such networks were extensively studied by Cover et. al. [2] in the 70's. Fostered by the increasing importance of ad hoc and wireless sensor networks, of late a great amount of valuable research has gone into relay networks. Data transmission with relay(s) not only raises the achievable rate of information transmission, but also provides alternative routes when the direct transmission is resourceconsuming or totally impossible. For wireless networks, the outage performance, defined as the probability of the instantaneous mutual information falling below a basic rate threshold, has been shown to improve dramatically due to the diversity gain offered by relay networks. This type of diversity has been named as cooperative diversity [3], which, as the word implies, is provided by the cooperation among various communication units. The authors of [3] suggested (amongst others) two simple relay schemes: Amplifyand-Forward (AF) and Decode-and-Forward (DF). Various other protocols such as compress-and-forward, estimate-and-forward etc. have been suggested in the literature as well. For a survey of these and other possible relaying protocols and their capacity results, see [4]. There has been a significant number of studies on optimal power allocation in wireless relay networks over fading channels. Optimal power allocation for information theoretic achievable rate maximization has been studied in detail in [5] (see also references therein), whereas [6] has studied the problem of optimal power allocation for outage probability minimization in relay networks. There have been parallel studies on optimal power allocation for signal-to-noise ratio maximization for single and multiple relay networks in [7] amongst many others.

While optimal power allocation can increase achievable rates or minimize outage probabilities specially with channel state information at the transmitter (and receiver), it is also critical to consider other important Quality-of-Service (QoS) criteria such as delay in designing a reliable wireless system, specially for voice/video communication as well as delay sensitive data communication applications in wireless ad hoc/sensor networks. In these applications, using lengthy codewords in order to capture the ergodicity of the

This work was supported by the Australian Research Council. CUBIN is an affiliated program of National ICT Australia (NICTA) fading channel and achieve the maximum expected throughput is not a useful approach. This has led researchers to consider various different notions of capacity of fading channels other than ergodic capacity - such as outage capacity and delay-limited capacity etc. A great survey on capacity notions for fading channels can be found in [8]. Delay constrained capacity optimization for wireless channels has been also a fruitful area of research. Following the seminal work of [9] where the average queuing delay was minimized by optimal power and rate control for data transmission over wireless fading channels, there have been further advances on optimal power and rate control for minimal average delay in [10]. Similarly, optimal power allocation for capacity maximization with coding delay using causal channel information was investigated using dynamic programming techniques in [11], and throughput maximization with both coding and queuing delay constraints has been studied in [12]. More recently, a stochastic power allocation method for expected capacity maximization with a finite coding delay constraint has been investigated in [13].

While there is a rich literature on optimal power control and scheduling with delay or deadline constraints for traditional wireless networks, the same cannot be said for cooperative wireless networks. Delay constraints have been considered as embedded in the concept of "effective capacity" for cooperative networks and optimal power allocation for such problems in [14]. Some preliminary investigations into cooperative transmission with queuing delay constraints have been made in [15]. However, there has been no in-depth study on power allocation for cooperative transmission with coding delay constraints where data transmission takes place over a finite number of blocks. This is indeed the focus of our paper. Similar to [11], we also impose the practical constraint that only causal channel information is available to the transmitting nodes. In particular, the main contribution of this paper is to address an optimal power allocation algorithm to maximize the expected achievable rate for a three node relay network with a pre-specified delay constraint (i.e, number of coding blocks) and a short-term (also known as peak) constraint on the total power (sum of source and relay power) over all blocks where only causal channel state information is available. As in [11], we also use Dynamic Programming (DP) based techniques for solving this problem. We evaluate the performance of the AF and DF schemes and compare their performances under delay and causality constraints with the standard direct transmission scheme. We illustrate via simulation results that cooperative transmission offers significant advantages over the direct transmission even with finite coding delay. The relative performances of the AF and DF schemes are also illustrated for varying available power levels and number of coding blocks. The benefits from using the optimal power allocation technique using DP methodologies are also illustrated in comparison with a sub-optimal technique that uses equal (sum of source and relay) power for all blocks. It is seen that in the low power regime, these benefits are much pronounced whereas in the high power regime, they are negligible for the $\mathrm{AF}$ and $\mathrm{DF}$ cooperative schemes. The effect of moving the relay from close to the source to close to the destination is also illustrated. Motivated the high computational demands of the DP based schemes, we also provide simple sub-optimal power allocation schemes for high and low power regimes, which are seen to be reasonably accurate via simulation 
studies.

The rest of the paper is organized as follows. Section II describes the general background and the network model and underlying assumptions used through the paper. In Section III-A, we study a case where there is only one block available for the transmission. We present a summary of the relevant results of optimal power allocation for the single block case which exist in various forms in the literature. DP based algorithms are then introduced to solve the multiple-block optimization problems in Section III-B. We propose the high and low SNR approximation based simple sub-optimal power allocation methods in Section IV. Extensive numerical results are provided in Section V.

\section{Problem Formulation}

We consider a three node cooperative relay network where the source sends data to the destination with the help of a relay node, as shown in Figure 1 and the relay does not produce its own data. It is assumed that the channel gains for all the three links- Source-Destination (S-D), Source-Relay (S-R), and, RelayDestination (R-D) - follow a block fading model. Within each block of data transmission, the channels are constant but they change from one block to another in an independent and identically distributed fashion. We do not assume that all the links are distributed according to the same distribution. In general, the statistical distributions of the channel gains of the three links can be different. In this paper, we will consider the three simple transmission schemes: Direct Transmission (DT), Amplify and Forward (AF) and Decode and Forward (DF). In the DT scheme, obviously the source transmits only directly to the destination, while in the AF and DF schemes, the source also uses the relay for data transmission. We assume a half-duplex time division transmission scheme where every transmission block (same as the block over which the channels remain invariant) is divided into two halves. During the first half, the source transmits to the relay and the destination. During the second half, the relay transmits to the destination and the source does not transmit. As assumed in [3], in the AF scheme, the relay only amplifies and forwards its received data to the destination. In the DF scheme, the relay first decodes and then forwards the decoded data to the destination. We assume that there is no queue at the relay node. We use $\gamma_{1}^{k}, \gamma_{2}^{k}$, and $\gamma_{3}^{k}$ to denote the channel states of the three channels during the $k$-th block of data transmission. We use the notations $\gamma^{k}=\left(\gamma_{1}^{k}, \gamma_{2}^{k}, \gamma_{3}^{k}\right)$ and $\gamma^{(k)}=\left\{\gamma^{1}, \gamma^{2}, \ldots, \gamma^{k}\right\}$. Clearly, $\gamma^{(k)}$ represents the causal channel state vector including all the channel state information (CSI) until block $k$ (inclusive). It is assumed that the destination has the exact knowledge of $\gamma^{(k)}$ and feeds this information back to the source and the relay through an error free feedback channel. Since the source and relay transmission powers are allocated based on this causal CSI, we denote the $k$-th block transmission powers of the source and relay by $P_{s}^{k}\left(\gamma^{(k)}\right)$ and $P_{r}^{k}\left(\gamma^{(k)}\right)$, respectively.

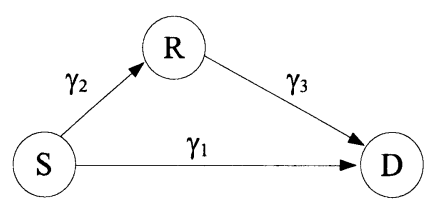

Fig. 1. A Relay Network with Random Fading Parameters

The coding delay constraint $K$ is usually determined by specific delay sensitive applications and is known before any information transmission takes place. The objective is to maximize the expectation of the achievable rate, and the total power (sum of source and relay powers) available over $K$ blocks is limited. In this paper, we will assume a short term power constraint which is sometimes also called a peak power constraint. In summary, the delay constrained capacity optimization problem with causal feedback is given as:

$$
\begin{gathered}
\max \mathbb{E}_{\gamma}\left[\sum_{k=1}^{K} C_{*}\left(P_{s}^{k}\left(\gamma^{(k)}\right), P_{r}^{k}\left(\gamma^{(k)}\right), \gamma^{k}\right)\right] \\
\text { s.t. } \quad P_{s}^{k}\left(\gamma^{(k)}\right), P_{r}^{k}\left(\gamma^{(k)}\right) \geq 0, \forall k \\
\sum_{k=1}^{K}\left(P_{s}^{k}\left(\gamma^{(k)}\right)+P_{r}^{k}\left(\gamma^{(k)}\right)\right) \leq K P_{0}
\end{gathered}
$$

where $P_{0}$ can be thought of as an average power constraint per block, and the function $C_{*}\left(P_{s}, P_{r}, \gamma\right)$ denotes the instantaneous mutual information of one of the three transmission protocols - DT, AF and DF respectively, which are given below:

$$
\begin{aligned}
& C_{\mathrm{DT}}\left(p_{s}, p_{r}, \gamma\right)=\log \left(1+\gamma_{1} p_{s}\right) \\
& C_{\mathrm{AF}}\left(p_{s}, p_{r}, \gamma\right)= \\
& \quad \frac{1}{2} \log \left(1+2 \gamma_{1} p_{s}+\frac{4 \gamma_{2} p_{s} \gamma_{3} p_{r}}{1+2 \gamma_{2} p_{s}+2 \gamma_{3} p_{r}}\right) \\
& C_{\mathrm{DF}}\left(p_{s}, p_{r}, \gamma\right)= \\
& \quad \frac{1}{2} \min \left\{\log \left(1+2 \gamma_{2} p_{s}\right), \log \left(1+2 \gamma_{1} p_{s}+2 \gamma_{3} p_{r}\right)\right\} .
\end{aligned}
$$

$\mathbb{E}_{\gamma}$ denotes the expectation operator with respect to the joint probability density function of the random variables $\left\{\gamma^{1}, \gamma^{2}, \ldots, \gamma^{K}\right\}$. Note also that in this paper, we may use the terms "expected capacity" and "expected maximum achievable rate" interchangeably in the context of relay networks, although strictly speaking this is not rigorous.

\section{The Optimal Power Allocation Schemes}

\section{A. Special Case - Single-Block Transmission}

Before we attempt to solve the optimization problem given by (1), it is useful to look at a simple case when there is only one block available for transmission, that is, $K=1$. It is obvious that in this scheme, the optimal direct transmission policy is to use the full power $P_{0}$ in the single block, and the corresponding expected capacity is given by $E\left[\log \left(1+\gamma_{1} P_{0}\right)\right]$.

In the case of cooperative transmission with the $\mathrm{AF}$ proto$\mathrm{col}$, the expected capacity maximization problem for $K=1$ amounts to maximizing $E\left[C_{\mathrm{AF}}\left(P_{s}(\gamma), P_{r}(\gamma), \gamma\right)\right]$ subject to the short term power constraint $P_{s}(\gamma)+P_{r}(\gamma) \leq P_{0}, P_{s}(\gamma) \geq$ $0, P_{r}(\gamma) \geq 0$. For the outage probability minimization case, the single block problem for the $\mathrm{AF}$ scheme amounts to minimizing $\operatorname{Pr}\left\{C_{\mathrm{AF}}\left(P_{s}(\gamma), P_{r}(\gamma), \gamma\right)<R_{0}\right\}$ subject to $P_{s}(\gamma)+P_{r}(\gamma) \leq P_{0}$, $P_{s}(\gamma) \geq 0, P_{r}(\gamma) \geq 0$. Note that here the dependence of the channel gains on the time index $k$ have been dropped since there is only a single block available for transmission. The corresponding optimization problems for the DF case can be formulated similarly. Solutions to these and related problems (usually with long term average power constraints) have been presented in various papers, for example, see [5], [14], [6] and references therein. For convenience, we briefly present the solutions to the above problems for the $\mathrm{AF}$ and DF schemes.

The basic idea behind solving the above optimization problems is to solve a corresponding instantaneous mutual information maximization problem with a short term sum power constraint (see [16] for details). The optimal power allocation policy obtained this way provides the solution to the expected achievable rate maximization problem.

It is easy to derive the optimal policy for the DF protocol due to convexity. The maximum achievable rate is given as

$$
R_{\mathrm{DF}}= \begin{cases}\frac{1}{2} \log \left(1+2 \gamma_{2} P_{0}\right) & \gamma_{1}>\gamma_{2} \\ \frac{1}{2} \log \left(1+2 \gamma_{1} P_{0}\right) & \gamma_{1} \leq \gamma_{2} \text { and } \gamma_{1}>\gamma_{3} \\ \frac{1}{2} \log \left(1+\frac{2 \gamma_{2} \gamma_{3}}{\gamma_{2}+\gamma_{3}-\gamma_{1}} P_{0}\right) & \gamma_{1} \leq \gamma_{2} \text { and } \gamma_{1} \leq \gamma_{3} .\end{cases}
$$

Due to the nonconcavity of the AF mutual information expression, it is more complicated to compute the optimal policy in this case. We 
would only present the final result because of the space limitation in this paper. The corresponding maximum achievable rate is

$$
\begin{aligned}
& R_{\mathrm{AF}}= \\
& \begin{cases}\tilde{C}_{\mathrm{AF}}\left(P_{r}^{*}, \gamma\right) & \gamma_{1}<\gamma_{3} \& P_{0}>\frac{\gamma_{1}}{2 \gamma_{2}\left(\gamma_{3}-\gamma_{1}\right)} \\
\frac{1}{2} \log \left(1+2 \gamma_{1} P_{0}\right) & \text { otherwise }\end{cases}
\end{aligned}
$$

where

$$
\begin{aligned}
& \tilde{C}_{\mathrm{AF}}\left(P_{r}^{*}, \gamma\right)= \\
& \frac{1}{2} \log \left(1+2 \gamma_{1} f\left(P_{r}^{*}\right)+\frac{4 \gamma_{2} f\left(P_{r}^{*}\right) \gamma_{3} P_{r}^{*}}{1+2 \gamma_{2} f\left(P_{r}^{*}\right)+2 \gamma_{3} P_{r}^{*}}\right)
\end{aligned}
$$

and $P_{r}^{*}$ is the unique solution of $f\left(P_{r}\right)+P_{r}=P_{0}$. Since the left hand side of the equation is monotonically increasing, one can easily achieve $P_{r}^{*}$ through bisection method and etc.

Since the above solution is quite complicated and an explicit expression for the capacity maximization is not available, a popular approximation (valid in the moderate to high signal-to-noise ratio (SNR) regime) is given by

$$
1+2 \gamma_{2} P_{s}+2 \gamma_{3} P_{r} \approx 2 \gamma_{2} P_{s}+2 \gamma_{3} P_{r}
$$

This approximation is widely used due to the fact that the resulting achievable rate expression for the $\mathrm{AF}$ case becomes concave jointly in $P_{s}$ and $P_{r}$. See for example [14] amongst many others. Excluding some details due to space limitations, the maximized achievable rate for the AF scheme with this approximation is

$$
\tilde{R}_{\mathrm{AF}}= \begin{cases}\frac{1}{2} \log \left(1+2 \gamma_{1} P_{0}\right) & \gamma_{1}>\gamma_{3} \\ \frac{1}{2} \log \left(1+\frac{2 u P_{0}}{1+u}\left(\gamma_{1}+\frac{\gamma_{2} u \gamma_{3}}{\gamma_{2} u+\gamma_{3}}\right)\right) & \gamma_{1} \leq \gamma_{3},\end{cases}
$$

where

$$
u=\frac{\gamma_{3}\left(\gamma_{1}+\sqrt{\gamma_{1} \gamma_{3}-\gamma_{1} \gamma_{2}+\gamma_{2} \gamma_{3}}\right)}{\left(\gamma_{3}-\gamma_{1}\right) \gamma_{2}} .
$$

Given the above optimal power allocation policies for a given channel realization, one can calculate the maximum expected capacity or the minimum outage probability of a single block transmission system by numerical methods or via Monte Carlo simulations, by averaging over a large number of simulated channel realizations. While exact expressions of expected capacity or outage probability are rare, there are high SNR approximations to the outage probability available in various papers such as [3], [17] etc.

\section{B. Optimal Power Allocation for Multi-Block Transmission}

In this section, we solve the original dynamic power allocation problems as proposed in (1) for the expected achievable rate maximization based on causal CSI, that is, based on past and current channel gains only. It should be obvious that for a given finite $K$, it is not possible to obtain the optimal power allocation for each block in closed form as in the case for $K=1$. In fact, even if all the channel states (including the future ones) are available, it is still quite difficult to calculate the maximum expected achievable rate for $\mathrm{AF}$ and $\mathrm{DF}$ cases (whereas the DT case can be solved by the well known water-filling algorithm) using constrained optimization techniques. This is simply because evaluating all possible combinations of conditions on channel triple $\gamma^{k}$, as in (4), (3) and (6) and obtaining the corresponding optimal power allocation is computationally prohibitive even for moderate values of $K$. This motivates us to use the dynamic programming methodology as also used in [11] for solving a delay constrained capacity optimization problem for the DT case over fading channels. Using dynamic programming techniques, the dynamic power allocation problem can be solved in $K+1$ stages. Starting at stage $K+1$, a sequence $\left\{S_{k}\right\}$ (which can be a function of transmission power) can be derived in a backward fashion. An algorithmic description of this method is described below in Algorithm 1. In this Algorithm, $C_{*}$ can possibly symbolize $C_{\mathrm{DT}}, C_{\mathrm{AF}}$, or, $C_{\mathrm{DF}}$ as shown in (2).

According to Theorem II.I in [11], the optimal solution to Problem (1) is given by $S_{1}\left(K P_{0}\right)$ for the expected capacity maximization

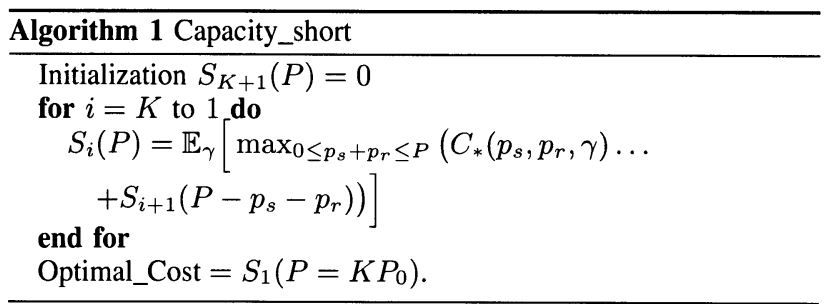

problem (see also Theorem III.I in [11] ) when $C_{*}=C_{\mathrm{DT}}$. It is straightforward to conclude the same for the $\mathrm{AF}$ and $\mathrm{DF}$ cases where the achievable rates are modified accordingly. We can now compute all the values of $S_{i}(P)$ where $i=1, \ldots, K+1$ and $0 \leq P \leq K P_{0}$, using Algorithm 1. In a practical system, such computations can be carried out at the destination node which is assumed to have the knowledge of all the channel gains. This $S$ matrix can then be fed back to the source and relay nodes and stored there. Upon feedback of causal CSI data, the source and the relay can then use another Algorithm 2 to allocate dynamically the powers for the current block. In the above algorithm, $R^{(i)}$ and $P^{(i)}$ denote the sum rate achieved

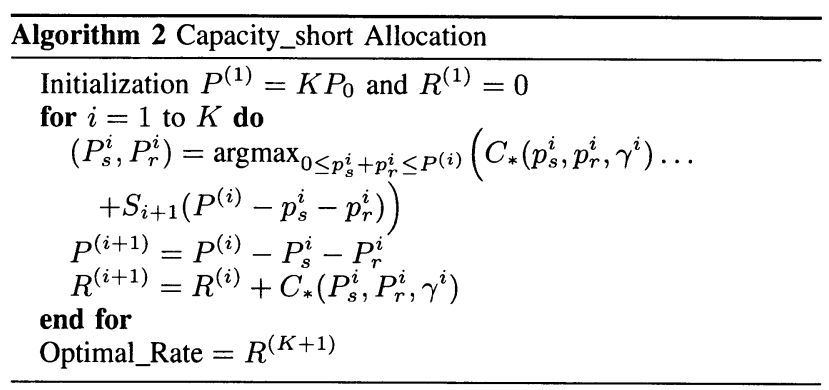

before transmitting block $i$ and the remaining power for blocks $i$ to $K$, respectively.

In summary, Algorithm 1 discretizes the power $P$ according to the computational capability of the destination node and the storage capability of the source and relay nodes. The 2-dimensional matrix $S$ is then computed and stored at the destination and forwarded to and stored in the source and relay nodes. When the system is online, as long as the current channel state information is available at the source and relay nodes, the system can allocate the transmitting power to the source and the relay by using Algorithm 2 instead. Since the channel state information is assumed to be i.i.d. over all blocks, the outage probability averaged over a large number of channel state instances should be very close to $S_{1}\left(K P_{0}\right)$, as long as $S_{1}\left(K P_{0}\right)$ is relatively accurate, which is equivalent to saying that the discretization is fine enough. The same conclusion obviously holds for the expected capacity maximization problem. We will have some further discussion on the discretization issues in Section $\mathrm{V}$ below.

\section{Simple Sub-optimal Power Allocation Schemes BASED ON HIGH AND LOW SNR APPROXIMATIONS}

In the last section, we solve the original problem with a multiblock coding delay by a DP based algorithm. However, this algorithm is computationally highly complex due to computation of the 2-D matrix $S$. Therefore, we adopt a reasonable assumption that when the CSI is only known for the current block and unknown for the future blocks (which are i.i.d), after assigning power to the current block, the system equally distributes the remaining power to the rest of the blocks [13]. As a result, we could divide the total available power into two parts: the power allocated to the current block $P^{i}$ where $P^{i}=P_{s}^{i}+P_{r}^{i}$ and the powers equally distributed to the rest of the blocks which are equal to $P^{k}=\frac{P^{(i)}-P^{i}}{K-i}$ where $i=1, \ldots, K-1$ and $k=i+1, \ldots, K$.

With a high SNR approximation for the AF protocol, we can 
approximate the achievable rate for those future blocks as follows

$$
\begin{aligned}
& \mathbb{E}_{\gamma}\left[\sum_{k=i+1}^{K} C_{\mathrm{AF}}\left(P_{s}^{k}, P_{r}^{k}, \gamma^{k}\right)\right] \\
= & (K-i) \mathbb{E}_{\gamma}\left[\frac{1}{2} \log \left(1+2 \gamma_{1}^{k} P_{s}^{k}+\frac{4 \gamma_{2}^{k} P_{s}^{k} \gamma_{3}^{k} P_{r}^{k}}{1+2 \gamma_{2}^{k} P_{s}^{k}+2 \gamma_{3}^{k} P_{r}^{k}}\right)\right] \\
\approx & (K-i) \mathbb{E}_{\gamma}\left[\frac{1}{2} \log \left(1+2 \gamma_{1}^{k} P_{s}^{k}+\frac{2 \gamma_{2}^{k} P_{s}^{k} \gamma_{3}^{k} P_{r}^{k}}{\gamma_{2}^{k} P_{s}^{k}+\gamma_{3}^{k} P_{r}^{k}}\right)\right] \\
\leq & \frac{(K-i)}{2} \log \left(1+2 \mathbb{E}\left[\gamma_{1}^{k}\right] P_{s}^{k}+\frac{2 \mathbb{E}\left[\gamma_{2}^{k}\right] P_{s}^{k} \mathbb{E}\left[\gamma_{3}^{k}\right] P_{r}^{k}}{\mathbb{E}\left[\gamma_{2}^{k}\right] P_{s}^{k}+\mathbb{E}\left[\gamma_{3}^{k}\right] P_{r}^{k}}\right) \\
\leq & \frac{(K-i)}{2} \log \left(1+\bar{c} P^{k}\right) \\
= & \frac{(K-i)}{2} \log \left(1+\bar{c} \frac{P^{(i)}-P^{i}}{K-i}\right) .
\end{aligned}
$$

The constant $\bar{c}$ in the last two lines can be derived from (6).

Through this approximation, the power allocated to the current block can be derived by solving a single variable optimization problem:

$$
\begin{gathered}
\max _{P^{i}} \frac{1}{2} \log \left(1+c^{i} P^{i}\right)+\frac{(K-i)}{2} \log \left(1+\bar{c} \frac{P^{(i)}-P^{i}}{K-i}\right) \\
\text { s.t. } \quad 0 \leq P^{i} \leq P^{(i)}
\end{gathered}
$$

where

$$
c^{i}= \begin{cases}\frac{2 u^{i}}{1+u^{i}}\left(\gamma_{1}^{i}+\frac{\gamma_{2}^{i} u^{i} \gamma_{3}^{i}}{\gamma_{2}^{i} u^{i}+\gamma_{3}^{i}}\right) & \gamma_{1}^{i} \leq \gamma_{3}^{i} \\ 2 \gamma_{1}^{i} & \gamma_{1}^{i}>\gamma_{3}^{i}\end{cases}
$$

and $u^{i}=\frac{\gamma_{3}^{i}\left(\gamma_{1}^{i}+\sqrt{\gamma_{1}^{i} \gamma_{3}^{i}-\gamma_{1}^{i} \gamma_{2}^{i}+\gamma_{2}^{i} \gamma_{3}^{i}}\right)}{\left(\gamma_{3}^{i}-\gamma_{1}^{i}\right) \gamma_{2}^{i}}$.

Using KKT conditions, it is easy to derive the optimal value

$$
P^{i^{*}}=\min \left\{\left[\frac{c^{i}+\frac{c^{i} \bar{c} P^{(i)}}{K-i}-\bar{c}}{c^{i} \bar{c}\left(\frac{1}{K-i}+1\right)}\right]^{+}, P^{(i)}\right\}
$$

Similarly, the power allocation scheme can also be derived to be the same formula (8) for the DF protocol with the only difference being

$$
c^{i}= \begin{cases}2 \gamma_{2}^{i} & \gamma_{1}^{i}>\gamma_{2}^{i} \\ 2 \gamma_{1}^{i} & \gamma_{1}^{i} \leq \gamma_{2}^{i} \text { and } \gamma_{1}^{i}>\gamma_{3}^{i} \\ \frac{2 \gamma_{2}^{i} \gamma_{3}^{i}}{\gamma_{2}^{i}+\gamma_{3}^{i}-\gamma_{1}^{i}} & \gamma_{1}^{i} \leq \gamma_{2}^{i} \text { and } \gamma_{1}^{i} \leq \gamma_{3}^{i}\end{cases}
$$

and $\bar{c}=\frac{2 \mathbb{E}\left[\gamma_{2}\right] \mathbb{E}\left[\gamma_{3}\right]}{\mathbb{E}\left[\gamma_{2}\right]+\mathbb{E}\left[\gamma_{3}\right]-\mathbb{E}\left[\gamma_{1}\right]}$ is

In the low SNR regime, the achievable rate for the future blocks

$$
\begin{aligned}
& \mathbb{E}_{\gamma}\left[\sum_{k=i+1}^{K} C_{\mathrm{AF}}\left(P_{s}^{k}, P_{r}^{k}, \gamma^{k}\right)\right] \\
& =(K-i) \mathbb{E}_{\gamma}\left[\frac{1}{2} \log \left(1+2 \gamma_{1}^{k} P_{s}^{k}+\frac{4 \gamma_{2}^{k} P_{s}^{k} \gamma_{3}^{k} P_{r}^{k}}{1+2 \gamma_{2}^{k} P_{s}^{k}+2 \gamma_{3}^{k} P_{r}^{k}}\right)\right] \\
& \approx(K-i) \mathbb{E}_{\gamma}\left[\frac{1}{2} \log \left(1+2 \gamma_{1}^{k} P_{s}^{k}+4 \gamma_{2}^{k} P_{s}^{k} \gamma_{3}^{k} P_{r}^{k}\right)\right] \\
& \approx(K-i) \mathbb{E}_{\gamma}\left[\gamma_{1}^{k} P_{s}^{k}+2 \gamma_{2}^{k} P_{s}^{k} \gamma_{3}^{k} P_{r}^{k}\right] \\
& =(K-i)\left(\mathbb{E}\left[\gamma_{1}^{k}\right] P_{s}^{k}+2 \mathbb{E}\left[\gamma_{2}^{k}\right] P_{s}^{k} \mathbb{E}\left[\gamma_{3}^{k}\right] P_{r}^{k}\right)
\end{aligned}
$$

Due to the fact that $P^{k}=P_{s}^{k}+P_{r}^{k}$ is small in low SNR situation, the achievable rate can be further approximated as

$$
\begin{aligned}
&(K-i)\left(\mathbb{E}\left[\gamma_{1}^{k}\right] P_{s}^{k}+2 \mathbb{E}\left[\gamma_{2}^{k}\right] P_{s}^{k} \mathbb{E}\left[\gamma_{3}^{k}\right] P_{r}^{k}\right) \\
&=(K-i)\left(\mathbb{E}\left[\gamma_{1}^{k}\right] P_{s}^{k}+2 \mathbb{E}\left[\gamma_{2}^{k}\right] P_{s}^{k} \mathbb{E}\left[\gamma_{3}^{k}\right]\left(P^{k}-P_{s}^{k}\right)\right) \\
& \leq_{P_{s}^{k}=P^{k}}(K-i)\left(\mathbb{E}\left[\gamma_{1}^{k}\right] P^{k}+2 \mathbb{E}\left[\gamma_{2}^{k}\right] P^{k} \mathbb{E}\left[\gamma_{3}^{k}\right]\left(P^{k}-P^{k}\right)\right) \\
&=(K-i) \mathbb{E}\left[\gamma_{1}^{k}\right] \frac{P^{(i)}-P^{i}}{K-i}=\mathbb{E}\left[\gamma_{1}^{k}\right]\left(P^{(i)}-P^{i}\right) .
\end{aligned}
$$

Formulating a similar optimization problem as in (7), we can show that for the low SNR regime

$$
P^{i^{*}}= \begin{cases}0 & \gamma_{1}^{i}<\mathbb{E}\left[\gamma_{1}\right] \\ P^{(i)} & \text { otherwise }\end{cases}
$$

which indicates that the system waits for a transmission block with a good channel and puts all the power into that block.

For the DF case when SNR is low, we can also apply a similar method. The power allocation scheme is

$$
P^{i *}= \begin{cases}0 & c^{i}<\frac{\mathbb{E}\left[\gamma_{2}\right] \mathbb{E}\left[\gamma_{3}\right]}{\mathbb{E}\left[\gamma_{2}\right]+\mathbb{E}\left[\gamma_{3}\right]-\mathbb{E}\left[\gamma_{1}\right]} \\ P^{(i)} & \text { otherwise }\end{cases}
$$

and $c^{i}$ is given by (9).

\section{Numerical Results}

In this section, we will provide a range of simulation results on the delay-constrained capacity maximization of the three node relay network using $\mathrm{AF}$ and $\mathrm{DF}$ schemes and compare their performances against the no cooperation (DT) schemes. We will present results where all links have no direct line of sight and undergo statistically independent Rayleigh fading (albeit with different means). For simplicity, all the simulations are based on a network where the relay node is located on the the straight line between the source and destination nodes.

We assume that the channel triple $\gamma=\left(\gamma_{1}, \gamma_{2}, \gamma_{3}\right)$ in the Figure 1 are exponentially distributed with means given by inverse of the $S-D$, S-R and R-D distances with a path loss factor of 4 . For computational simplicity, the S-D distance is normalized to 1 and, furthermore, the relay node is assumed to be at a distance of $d_{\mathrm{S}-\mathrm{R}}=0.4$ unless otherwise stated.

As we mentioned before, the resolution of the discretization has substantial effects on the performance of the DP algorithm. When the numbers of discretized power and channel-gain levels are large enough, the value calculated via Algorithm 1 is consistent with the one obtained via Algorithm 2 which averages achievable rates over a large number of random channel realizations. However, when the computational/storage capability is limited (smaller number of discretization levels), the size of the $S$ matrix is limited and the values of $S_{1}\left(K P_{0}\right)$ are not as accurate. The results obtained by averaging over a large number of channel realizations, in this case, are relatively more accurate. It should be obvious that in the case of cooperative transmission, the number of discretization levels required for reasonably accurate calculations of the relevant $S$ function is much higher due to the increased dimensionality of the CSI space. Thus the inaccuracy of the $S$ function inevitable due to limited computational resources will be more apparent in the cooperative transmission case. Therefore, in what follows, the expected rates achieved by the various power allocation schemes are calculated by averaging over 100000 channel realizations.

In order to illustrate the effectiveness of the DP algorithm, we compare the performance of the DP based power allocation against the performance obtained by using equally distributed (ED) total source and relay power $P_{0}$ for each block. Figure 2 clearly shows this improvement obtained (normalized by total power spent) in maximizing the expected achievable rate $\frac{C_{D P}-C_{E D}}{K P_{0}}$ for various values of $K$ at different power levels. Note that the improvement is considerable in the low power regime, for example, for $P_{0}=0.05$, this improvement can be almost doubled (for the AF case) as we increase $K$ from 2 to 14 . As the power level $P_{0}$ is increased, the improvement obtained by using the DP algorithm decreases in general. Interestingly, while DT seems to still maintain a reasonable improvement at a high power level $\left(P_{0}=1.0\right)$, the improvements for $\mathrm{AF}$ and DF become negligible, which means that there is not much to gain by using the DP algorithm compared to equally distributing the total power among all blocks for the AF and DF cooperative transmission schemes in the high power regime, i.e. the optimal solution is inclined to equally distribute power to each block. 


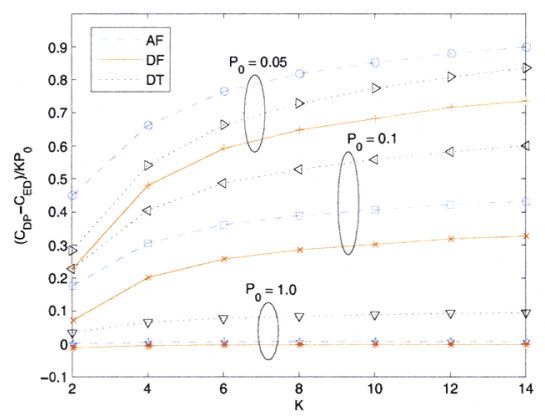

Fig. 2. Expected Capacity Improvement per unit power (via DP) versus Various Coding Delay $\mathrm{K}$

In addition, some results of the expected capacity (or achievable rate for the AF and DF schemes) achieved by Algorithm 2 are given in Figure 3. In order to achieve a fair comparison between DP and ED algorithms, the capacity gain obtained by the DP algorithm is normalized by $K P_{0}$ as we did in Figure 2. Figure 3 demonstrates that both $\mathrm{AF}$ and $\mathrm{DF}$ scheme achieve more optimistic result than DT and the DF scheme dominates when the S-R distance is short. However this advantage is no longer valid when the relay gets close to the destination $-d_{\mathrm{S}-\mathrm{R}}=0.8$.

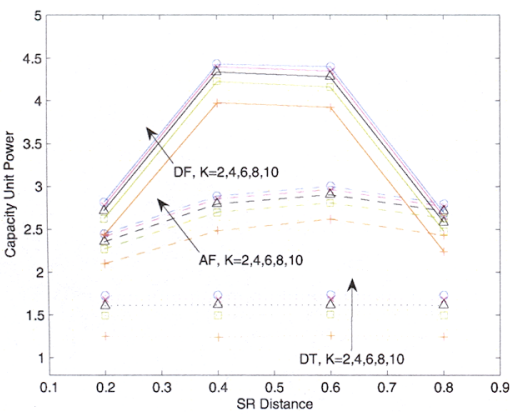

Fig. 3. Normalized Expected Capacity for DT, AF and DF, Various SR Distances When $P_{0}=0.05$

Finally, Figures 4 and 5 present the rates achieved by the simple sub-optimal schemes we proposed in Section IV. All the solid lines symbolize the results achieved by the DP based algorithm. As it can be seen, the two proposed approximation schemes perform well in the high and low SNR regime respectively. However, it seems that in the low SNR range of the DF case the low SNR and the high SNR based algorithms perform equally well. The explanation is that when $P^{(i)}$ in (8) is small the optimal value for high SNR method can be out of the range of $\left[0, P^{(i)}\right]$. As a result, this high SNR approximation degenerates to the low SNR scheme, i.e. if the channel information passes a threshold it puts all the power to the current block; otherwise, it waits until a more optimistic channel block appears.

\section{REFERENCES}

[1] E. C. van der Meulen, "Three-terminal communication channels," Adv. Appl. Prob., vol. 3, pp. 120-154, 1971.

[2] T. M. Cover and A. E. Gamal, "Capacity theorems for the relay channel," IEEE Trans. on Inform. Theory, vol. IT-25, no. 5, pp. 572584, Sep. 1979.

[3] J. N. Laneman, D. N. C. Tse, and G. W. Wornell, "Cooperative diversity in wireless networks: Efficient protocols and outage behavior," IEEE Trans. on Inform. Theory, vol. 50, no. 12, pp. 3062-3080, Dec. 2004.

[4] V. Stankovic, A. Host-Madsen, and Z. Xiong, "Cooperative diversity for wireless ad hoc networks," IEEE Signal Processing Magazine, pp. $37-49$, September 2006

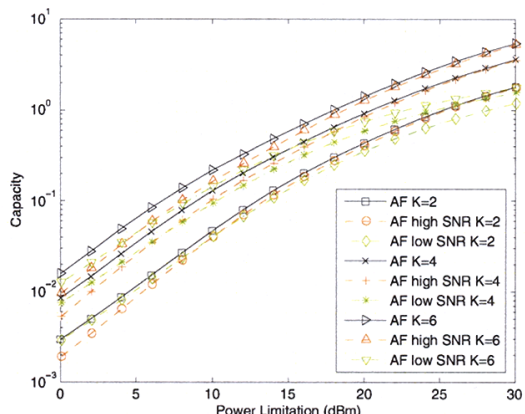

Fig. 4. Sub-optimal Power Allocation with High and Low SNR Approximations for the AF Scheme

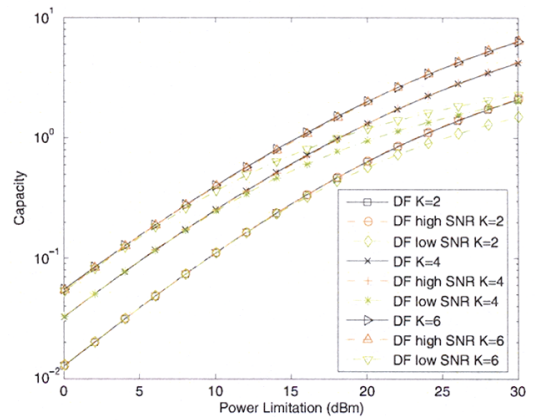

Fig. 5. Sub-optimal Power Allocation with High and Low SNR Approximations for the DF Scheme

[5] A. Host-Madsen and J. Zhang, "Capacity bounds and power allocation for wireless relay channels," IEEE Trans. on Inform. Theory, vol. 51, no. 6, pp. 2020-2040, June 2005.

[6] N. Ahmed, M. A. Khojastepour, and B. Aazhang, "Outage minimization and optimal power control for fading relay channel," in IEEE Inform. Theory Workshop (ITW), San Antonio, TX, USA, Oct. 2004, pp. 458462.

[7] Y. Li, B. Vucetic, Z. Zhou, and M. Dohler, "Distributed adaptive power allocation for wireless relay networks," IEEE Transactions on Wireless Communications, vol. 6, no. 3, pp. 948-958, March 2007.

[8] E. Biglieri, J. Proakis, and S. Shamai, "Fading channels: Informationtheoretic and communications aspects," IEEE Transactions on Information Theory, vol. 44, pp. 2619-2692, October 1998.

[9] R. Berry and R. Gallager, "Communication over fading channels with delay constraints," IEEE Transactions on Information Theory, vol. 48, no. 5, pp. 1135-1149, May 2002.

[10] I. Bettesh and S. Shamai, "Optimal power rate control for minimal average delay: The single-user case," IEEE Trans. on Inform. Theory, vol. 52, no. 9, pp. 4115-4141, Sep. 2006.

[11] R. Negi and J. M. Cioffi, "Delay-constrained capacity with causal feedback," IEEE Trans. on Inform. Theory, vol. 48, no. 9, pp. 24782494, Sep. 2002.

[12] N. Ahmed and R. Baraniuk, "Throughput maximization for arq-like systems in fading channels with coding and queuing delay constraints," in Proc. 38th Asilomar Conference on Signals, Systems and Computers, November 2004, pp. 1463-1467.

[13] K.-K. Wong, "Stochastic power allocation using causal channel information for delay-limited communications," IEEE Communications Letters, vol. 10, no. 11, pp. 748-750, November 2006.

[14] J. Tang and X. Zhang, "Cross-layer resource allocation over wireless relay networks for quality of service provisioning," IEEE JSAC, vol. 25, no. 4, pp. 645-656, May 2007.

[15] H. Wang, "Cooperative transmission in wireless networks with delay constraints," in Proc. Wireless Communications and Networking Conference, March 2004, pp. 1933-1938.

[16] G. Caire, G. Taricco, and E. Biglieri, "Optimal power control over fading channels," IEEE Trans. on Inform. Theory, vol. 45, no. 5, pp. 1468-1489, July 1999.

[17] A. Ribeiro, X. Cai, and G. Giannakis, "Symbol error probabilities for general cooperative links," IEEE Transactions on Wireless Communications, vol. 4, no. 3, pp. 1264-1273, May 2005. 